\title{
1 Assessment of some issues in CL-theory and program development
}

\author{
Danny De Schreye and Marc Denecker \\ Departement Computerwetenschappen, Katholieke Universiteit Leuven \\ Celestijnenlaan 200A, B-3001 Heverlee, Belgium \\ email \{dannyd,marcdn\}@cs.kuleuven.ac.be
}

\begin{abstract}
We make an assessment of the area of theory and program development in Computational Logic. We point out what we believe to be main causes for success or failure in this area. We revisit the "Algorithm = Logic + Control" equation of Kowalski and show how we believe it could be better understood and used. We indicate some promising directions for further work.
\end{abstract}

\subsection{Introduction: on assessment}

The area can be assessed with respect to different lines of objectives. The first is with respect to its achievements in fundamental or basic research. The second with respect to its impact on the software market.

\subsubsection{Fundamental research}

Again, one could distinguish between two lines of criteria. On the highest level, a very general criterion for the quality of basic research is scientific elegance. From two solutions to a same problem, criteria for fundamental research should select the one with the highest conceptual elegance. With respect to this, approaches to program and theory development in Computational Logic have performed exceptionally well.

However, an assessment could also be done with respect to the more specific long term objectives of this research area. These have often been stated by many different people of our community. As such, the line of thought for the long term objectives stated below is by no means original.

The motivations are rooted on two key observations. One is that software development is still very much a hand craft, in which each statement, each line of code, needs to understood in order to develop, adapt and maintain programs. The other observation is that, unlike other disciplines in engineering - such as chemical or mechanical engineering - there are very few formal mathematical foundations that can be exploited to support the software engineering process in a foundational and rigid way.

What is badly needed are techniques that bring software development from a hand craft to a scientifically supported, semi-automated process. In order to achieve this, since program development requires the understanding 
of each bit of code, the tools that provide the support for semi-automatization need to be semantics-based. In particular, we need formal, semantics-based tools for specification, analysis, verification and optimization of software.

This in turn imposes strong requirements on the programming languages for which such development can take place. In the context of programming languages with a very complex formal semantics, the realization of the mentioned semantics-based tools would be extremely difficult, if not impossible. Declarative languages, including Logic Programming languages, through their simple formal semantics, provide an ideal setting in which these long term objectives can be achieved.

\subsubsection{Impact on the software market}

Our perception of the software market is that it has two main characteristics that very much influence the potential impact of new technologies. The first of these is that the software market is inherently conservative, in the sense that it cannot, and should not, invest strongly in unstable technology. There are some exceptions to this. In particular, stand-alone applications, urgent problems for which no alternative solutions exist, or major-vendor supported hypes form such exceptions.

To clarify how this affects Computational Logic's impact in particular, we want to mention the example of the University Hospital at Leuven. Over the last 10 years, this very large hospital (with its several thousands of beds, one of the bigger world-wide) has been using Prolog as the basis for its information system, both for administrative and medical applications. As such, it has probably been one of the largest Prolog users in the world. Due to bankruptcy of their Prolog vendor, they were faced with the problem of finding a reasonably compatible Prolog at a reasonable price. This turned out to be difficult. So, considering the lack of guarantee on the stability of a potential new vendor (and the desire to move towards much more distributed and Internet based systems), they decided to move to Java. The example shows that our technology is insufficiently stable for companies to rely on it for their critical systems.

A second characteristic of the software market is that its demands on technology evolve very fast. Much faster than the speed at which basic research can produce solutions. In fact, computer science in general is much more driven by the demands imposed by industry than by previous progress achieved in fundamental research. As a result, few fundamental solutions to posed problems emerge sufficiently timely in order to be relevant, and most often ad-hoc partial solutions are preferred and become widely accepted.

At the Strategic meeting of the European Compulog Network of Excellence, held in Rome in the summer of 1997, a proposal was made to enhance the impact of Computational Logic on the software market by focusing on Internet-related research and applications (see [17]). 
Although this is an interesting idea, we do see some problems with it. We do not believe that from the outside world Computational Logic is perceived as a paradigm which is specifically targeted towards (and suitable for) Internet development. Moreover, Computational Logic languages have definitely not been designed to deal with the specific issues, such as security, distributed programming, updates and component-based programming, that this application domain requires. Competing languages, like Java, do have specific support for these issues. As such, it is unclear whether it is realistic to believe that we can compete, and that Computation Logic will indeed acquire a part of this market.

\subsubsection{Initial conclusions and overview}

At least in Japan and Europe, Computational Logic has had the status of a minor hype around 1990. In Europe in particular, the Esprit research program funded quite a few projects, both in basic research and in R \& D. Note that Esprit is a framework for industry-related research and therefore its funding of Computational Logic necessarily expresses industrial interest. In retrospect, one could criticize a majority of these projects for having focussed too much on basic research issues, while devoting less attention to the more practically relevant issues. Still, there have been important breakthroughs in the areas of Constraint Logic Programming and Inductive Logic Programming, with specific niches of application domains. In general, restricted to the area of program and theory development, the promises made by this area seemed to be too long-term to keep the interest from the outside world vivid.

In the remainder of the paper, we assess different areas related to theory and program development in Computational Logic. In particular: language implementation, program analysis and program transformation. We then discuss a novel view on the famous 'Algorithm $=$ Logic + Control' equation of Robert Kowalski ([11]), which played an important role in theory and program development in the past. We then briefly comment on software engineering (or the lack of it in Computational Logic) and conclude with some promising future directions.

\subsection{Implementation, analysis and transformation}

\subsubsection{Implementation}

Within Logic Programming, language implementation has possibly been one of the most successful areas (see [20]). Around the beginning of the eighties, this area achieved highly efficient, industrial quality implementations for standard Prolog. Later, in the beginning of the nineties, similar results were obtained for Prolog augmented with delaying execution mechanisms ([16]), and by the end of the nineties, also for several extensions with constraint 
solving ([9]). Quoting Bart Demoen, industrial quality, efficient implementations can be expected for further extensions, including tabulation (see e.g. [26]) and abduction ([10]), in the coming years.

Another exponent of the work in this area is the success of Mercury (see [24]), where due to certain language restrictions and program declarations an efficiency comparable to C (and sometimes better) is obtained. Yet another, be it indirect success, is that some of the implementation techniques developed for Prolog, specifically on the level of memory management and garbage collection, were adopted in the implementation of Java (actually, even some of the implementors were imported).

A main strong point that boosted the achievements in this area is that language vendors actually invested in it. This is in contrast to some other areas of work, where no such support was given. We will return to this point below. A main weak point is that we never reached a highly efficient, industrial quality public domain Prolog. Having such a 'standard' Prolog available would have very much facilitated and boosted the work on the development of tools. It would have allowed much easier evaluation, comparison, integration and cross-fertilization between various techniques, which are all of the utmost importance in order to lift the techniques to wide-scale usability.

\subsubsection{Analysis and transformation}

In this section, we briefly assess achievements of Computational Logic in such areas as abstract interpretation, assertion-based analysis, termination analysis, unfold/fold transformation, partial evaluation and deduction, and program specialization in general. We refer to the tenth anniversary issue of the Journal of Logic Programming ([3]) for surveys on these areas. On the whole, the work in these areas has delivered much high quality basic research. Also, many good prototype systems have been developed. However, very few of these prototypes have been integrated into industrial quality Logic Programming languages.

What have been the bottlenecks for achieving such integrations? A first and important one are the non-declarative features of Prolog. Although the very existence of a practical, efficiently implemented programming language, such as Prolog, has been of the utmost importance for our community, many powerful semantics-based analysis and optimization techniques that were developed for pure logic programs failed to scale up well for full Prolog. In some cases, the extensions to full Prolog became extremely messy and lost all the elegance of their "pure" counterpart. In most cases, the extended techniques simply lost most of their precision and power, failing to accurately treat the non-declarative features.

Similar bottlenecks turned up in the context of extending techniques from definite programs to normal ones, and from left-to-right SLD-resolution to the treatment of more complex computation mechanisms, e.g. those dealing with delay mechanisms (e.g. [16]) and constraint solvers (e.g. [9,18]). In both 
of these cases, somewhat similar to the extension to full Prolog, the cause of the bottleneck is the increased complexity of the semantics. This refers back to the statement in the introduction, that languages with a simple semantics are essential to make the development of semantics-based development tools feasible.

The semantics of logic programs with negation has been a matter of discussion and controversy for a long time. Although most issues have been resolved ([1]), the resulting preferred semantics are by no means simple. As a result, the percentage of works on program development that restrict the attention to definite programs is extremely high. Moreover, depending on the specific sub area, those techniques that do treat negation often only provide weak results for negative goals. For instance, most partial deduction methods only allow unfolding of negative literals when these are ground at specialization time (which is very restrictive due to the very nature of partial deduction).

In the context of more involved computation mechanisms, including delay mechanisms and constraint solvers, the increased complexity is on the level of the procedural semantics. Here again it turned out to be very hard to scale up techniques to these more complex semantics, while preserving elegance and precision. For instance, termination analysis under coroutining computation rules has taken much work and has produced fairly weak results. Similarly, abstract interpretation of constraint logic programs tends to loose a lot of precision.

Another reason for the lack of integration of development techniques in industrial quality systems is the weak position of the vendors. In contrast to the area of language implementation, vendors have not been able to support the research community in the integration of development tools into their systems.

A final bottleneck is that, at least for some of the subareas, there has been insufficient implementation oriented work in this area. In part this is due to a strong interest in theoretical issues, which is present in many areas in our field. Another important cause is that requirements for publications on implementation oriented work seem to be demanding a larger workload than for theoretical work. Most often, the implementations alone already require a substantial amount of work. In addition, requirements on detailed experimentations and comparisons with other approaches leads to an unbalance between the workloads attached to publication on practical versus theoretical work. This seems unreasonable. A change in attitude of the referees and editors is desirable to undo this.

\subsubsection{The need for integrated development tools}

Several semantics-based development techniques require similar types of information as input. Modes, types, data- and program flow patterns are of use for different verification and optimization techniques. This suggests that 
work should aim at developing integrated analysis/verification/optimization tools, in which the same information could be exploited in different ways. At the very least, this would solve efficiency problems, relaxing the precision/efficiency trade-off.

Developments in this direction have been started in the last few years in different contexts. For instance, integration of abstract interpretation in termination analysis and in program specialization has received increasing attention. An integrated analysis tool, linked to an optimization tool, is under development in Madrid $([8,19])$. Also, in the Mercury language, various types of information, partly declared, partly inferred, are combined in a powerful way to do both optimization and verification ([24]).

Taking this a step further, it has been suggested by Maurice Bruynooghe to further integrate these tools in a programming language specific editor. On the lowest level, the editor could support syntactic verification. Combined with incremental analysis, it could provide semantic verification. On top of that, based on the results of analysis, interactive, semi-automated optimization could be performed. In the context of open-box constraint logic programming, this could be enhanced even further. Today's open-box CLP languages ([18]) offer very rich control declaration languages. Combined and integrated with editing, analysis, verification and optimization support, such flexible control declaration languages could provide development tools that are in many ways similar to powerful program synthesis systems, such as the KIDS system of D. Smith ([23]).

\section{3 "Algorithm = Logic + Control" revisited}

The "Algorithm = Logic + Control" equation of [11] has motivated much work related to program development, especially in the context of program transformation and synthesis. In recent years, Robert Kowalski has revisited the equation, expressing that one should stress much more the procedural reading of logic programs, while reducing the emphasis on the declarative (in particular, the model theoretical) one ([13]).

As an initial response to that, we cannot help wondering why Logic Programming would be better at expressing procedures than procedural programming languages themselves. In some cases, in which the procedures are given in terms of natural language sentences (such as in legal reasoning applications), logic programs could indeed be the more natural way to encode such procedures. In many other cases though, such as in expressing algorithms, procedural languages seem to be more fit. This observation is backed up by many in our community. It suffices to inspect the literature to check how algorithms are typically represented in Logic Programming publications. The preferred language tends to be a Pascal-like one. Only in exceptional cases, when the author wants to illustrate his Prolog code for some algorithm, a Logic Programming style specification is used. 
In this section, we want to revisit the equation from a different angle. We want to point out how the equation has led to problems in the design of logic programs in the past, especially in the context of software engineering criteria, and we want to indicate some directions for solutions.

\subsubsection{A Pandora box called $\mathrm{A}=\mathrm{L}+\mathrm{C}$}

Let us first restrict the control, $C$, to SLD(NF)-resolution and afterward extend the discussion to more complex computation mechanisms. In this restrictive context, $A=L+C$ has created the conviction (or illusion) that we can simply represent a problem domain using declarative Horn clauses, after which SLD(NF) can generate solutions to particular queries. Practice has shown that this only works for very simple problems. As soon as a problem needs the traversal of larger search spaces, or needs other problem solving techniques than mere deduction, the declarative, Horn clause based approach is insufficient.

One may justly argue that the above statement seems to be contradicted by the many (pure) Prolog applications that have been developed in practice. Even most of the applications developed using the non-pure features of Prolog could probably also have been developed successfully without these non-pure features, at the expense of some efficiency.

The problem is that the Prolog programming used in such applications may be declarative in the formal mathematical sense (meaning: having a clear model-theoretical semantics), but it is not declarative with respect to the criteria that a software engineering methodology would impose.

In particular, successful larger Prolog applications are based on term-based programming. In term-based programming, the knowledge on the problem domain is not coded in atoms and clauses, but using terms. These terms are then grouped into lists, which actually represent theories of knowledge. Finally, Horn clauses are used to define new problem solvers, richer than SLD(NF)-resolution, which manipulate the theories encoded in the lists and which themselves are executed under SLD(NF).

As such, in term-based programming, the basis of the development is an equation $A=L^{\prime}+C$, where $\mathrm{L}^{\prime}$ is not the logic of the problem domain, but a logic defining some other execution mechanism, richer than SLD(NF), together with the original knowledge grouped into lists.

Now, the reason why this can be referred to as a Pandora box, is that in this representation, the knowledge on the problem domain does not appear as a first class citizen in the representation. The domain knowledge is buried inside lists, hidden inside procedures in $L$ '. As a result, none of the usual requirements of software engineering, like re-usability, maintenance, etc. of that knowledge is achieved. If several applications are developed based on the same knowledge domain, each program will store and manipulate its own copy of the same data, with the usual disadvantages of duplication resulting from that. 
Of course, a simple way to solve this is to encode the logic $L$ in a separate theory, and to write a meta-program, $M$, such that execution of $L$ under $M$ under SLD(NF) is the same as executing $L$ ' under SLD(NF). In the resulting equation, $A=L+M+C$, the domain knowledge is now explicitly represented and accessible. Partial evaluation of $L$ and $M$ could then yield $L$ 'again, which could be considered as a more efficient, lower level implementation.

\subsection{2 "AlgorithmS = Logic + ControlS"}

The discussion above reveals a deeper conflict between the view of knowledge (or software) engineering on the one hand, with the traditional view of Logic Programming on the other hand. In knowledge engineering it is considered vital to have the knowledge base designed independently from the problem solving strategies used. Moreover, this implicitly assumes that a variety of different problem solvers can act on the same knowledge, since it is practically impossible to have one single solver that can (efficiently) solve a variety of problems on that same knowledge. In particular, one might provide deductive, abductive, inductive, constraint based, model generator based and simulator solvers, all available in the same environment and acting on the same knowledge base to solve different problems.

In Logic Programming, the traditional view has been to have one specific procedural interpretation, based on SLD(NF)-resolution, to solve all types of queries. The very name of "Logic Programming" reflects its focus on a fixed procedural interpretation for the given logic formulas, as the notion of "programming" can only exist if the execution model is known (and taking into account) in the development of the programs.

Some people have tried to bridge the gap between these two views by promoting program synthesis and transformation as a means to derive efficient programs from purely declarative specifications. Although this work has had some successes (see e.g. [5,6]), it is commonly believed that optimally efficient algorithms cannot be derived automatically from specifications. Note that the KIDS system ([23]) is not a counter example to this statement, since this system requires interaction with the programmer.

Turning back to the aspect of having a variety of different solvers available for solving different problems concerning a same knowledge base, it is interesting to note that this is exactly what the evolution on execution models for logic programs has been achieving over the last decennium. The introduction of delays, of various types of constraint solvers, in particular the open-box constraint solvers which provide an entire spectrum of different solvers combined in one, of tabulation ([26,21]), of model generation ([14]), of abductive ([10]) and inductive solvers $([15])$, already provide a rich problem solving toolkit to support the knowledge engineering approach. However, as far as we know, current systems do not integrate a significant number of the above solvers into one powerful development system. We will return to this issue in the conclusions. 
All the above suggest a new equation, "AlgorithmS = Logic + ControlS", that stands as a basis for Computational Logic theory and program development for the beginning of the next century. According to this equation, the logic of a problem domain is modeled in a theory $L$, which is reused for every possible problem one aims to solve within the domain. Depending on the nature of the specific problem at hand, an appropriate problem solver is selected, giving rise to a corresponding algorithm. The selection of the solver may be a hard task, especially when the variety of available techniques continues to increase. In addition, the resulting algorithms may be further optimized through analysis and specialization. If the knowledge base needs to be modified, the selection of the appropriate solvers may need to be revised and the optimization phase will definitely need to be reexecuted.

Whether within this picture there is still room or need for a (manual) implementation phase would depend on the complexity of the application. With the above approach we have only boosted the complexity of the type of problems which we can solve in a naive $A=L+C$ way. Undoubtably, this will not exactly get us quick-sort from a naive generate-and-test-sort specification (although, by selecting the appropriate constraint-based pruning and enumeration techniques, additionally optimized through specialization, we might get something which is close enough for our purpose!). So, in some very time-critical applications, an implementation phase will be needed. In such a phase, the logic $L$ would still need to be remodeled to a logic $L$ ', more suitable for the particular problem. However,

1. the logic $L$ should remain central in the system, as a basis for future modifications and reuse, while $L$ 'is only considered as a low level implementation for the specific problem,

2. the implementation step from $L$ to $L$ ' should be very well documented, so that future maintenance, restarting from $L$ is not endangered.

In [4], Alan Bundy gives a different critical evaluation of the "Logic $=$ Algorithm + Control" equation. Similar to the discussion above, he also argues that, in general, a naive use of the equation can be lead to unacceptably inefficient programs. Different from us, he argues to extend the expressivity of the logic and to use program synthesis methods to transform richer logical specifications into efficient Horn programs. We believe that this is a valid alternative, but that, most likely, human interventions will continue to be required to guide such synthesis methods.

Also related is [12], where Robert Kowalski argues that Logic Programming needs to be extended with abduction and meta level reasoning to be able to use logic for all aspects of computation. The extension with meta level reasoning is in agreement with our concluding comments in Section 3.1. We see the extension with abduction as just one example of the many additional computation mechanisms that would be useful to increase the problem solving power of standard Logic Programming. 


\subsubsection{Software engineering}

One issue that has not been raised above is how to design the logic $L$. We are not aware of the existence of a methodology for analysis and design in the context Computational Logic. On the other hand, Object-oriented analysis and Object-oriented design provide methodologies that are by now widely accepted in software engineering practice.

To our surprise, we noticed that in some of the approaches to OO-analysis (e.g. [2]), one of the main claims on the benefits of the approaches is that they provide fully declarative specifications of the analyzed problem domains. What is meant here is that the domains are entirely described in terms of objects, attributes and constraints.

It seems extremely important to connect Computational Logic theory and program development to such methodologies for analysis and design. Considering the declarative nature of some of these approaches, a push-button conversion between the OO-based representations and their logic counterpart should be very feasible.

\subsection{Conclusions: future directions}

\subsubsection{Integration}

Over the years, work in Logic Programming has spread out into a large number of increasingly less connected subareas. Some of the most prominent are formal semantics, non-monotonic reasoning, language implementation, program analysis, program synthesis and transformation, constraint logic programming, concurrent logic programming, functional-logic programming, abductive logic programming, inductive logic programming, deductive databases, natural language and logic programming, internet related developments and multi-agent related developments. For many of these areas, recent new research contributions often provide further extensions to techniques, methods, implementations and theory which already reached a high degree of refinement and specialization in the past. As a result, it often takes considerable expertise in a specific sub area to appreciate the significance of new contributions. This in turn has motivated the different subareas to organize their own specialized meetings, workshops and conferences, disconnecting from the main general conferences in the field.

Very much work remains to be done on integrating the key results obtained in these different subareas. By way of example, constraint logic programming has achieved impressive results on certain classes of problems. On the other hand, tabulation, more recently introduced in the area of deductive databases, also achieves very efficient problem solving on a variety of problems. As far as we know, the integration of constraint solving with tabulation, and their combined potential for applications, has not been studied at all. 
One could produce a very long list of technological and theoretic achievements in Computational Logic, similar as in the example above, that have received considerable attention in isolation, but none in the context of other achievements. As a result, current Logic Programming languages only incorporate a fraction of the knowledge representation and problem solving power that the field produced in its various subareas over the last decennium. Also, formal theories only clarify the semantics and properties of isolated fragments of the full richness of the paradigm.

It is likely that many of the established techniques and results from separate subareas simply cannot be fruitfully and elegantly combined. But in many cases, the question of whether integration is feasible and useful has not received proper attention.

We believe that such integration activities are very important for the future of the field. In the areas involved with program and theory development in particular, they are crucial to obtain the long term objectives, as expressed in the introduction.

Of course, these problems are hard. They require a broad background, including expertise from different subareas. As such, they require a strong involvement from senior researchers in the field, and/or tight cooperation between different research teams.

At K.U.Leuven, a new research project entitled "LP+: a second generation Logic Programming language" was recently started to address some of these integration issues. It includes aspects on integration of knowledge representation in open theories, abduction, constraint solving, tabulation, efficient implementation, program and theory development techniques, efficient integrity checking and learning. We refer to [22] for an overview.

\subsubsection{Computational Logic as a research vehicle for Computer Science}

Another interesting direction for future research in Computational Logic lies in exploring the possibilities of porting and applying tools and techniques developed in Computational Logic to other programming paradigms. In the past, insufficient attention was given to applicability of methods and techniques outside the field. In particular, due to the powerful language features in the paradigm, such as unification, resolution and backtracking, certain problems, like for instance program optimization and analysis, are viewed upon differently from the Logic Programming perspective than from the perspective of languages with less powerful constructs. This sometimes leads to the development of richer techniques in Logic Programming, of which the applicability is not necessarily restricted to Logic Programming.

A typical example is positive supercompilation. Supercompilation ([25]) is a program transformation technique developed for functional programming languages, which extends partial evaluation. It is based on driving instead of 
reduction, where (in positive supercompilation) driving essentially generalizes matching to unification, introducing non-determinism in the unfolding. It has been shown in [7], that positive supercompilation is exactly the result of applying partial deduction to functional programs. As a side effect, the complex issue of how to control positive supercompilation could be resolved by importing results from Logic Programming on the control of partial deduction.

The example is by no means a stand-alone case. Other examples are for instance the way in which the apparently very different transformation techniques of tupling and deforestation in Functional Programming become very similar by moving from a functional to a relational representation. Yet another example, in the context of language implementation, is how memory management techniques for Prolog were adapted to the implementation of Java.

Aside from the powerful language features, applying techniques developed in Computational Logic to other paradigms is also very much facilitated by the fact that logic programs are so well suited for manipulating programs as data.

\section{Acknowledgement}

We shamelessly stole ideas from Bart Demoen on the assessment of language implementation, and from Maurice Bruynooghe on future directions for development tools. We are greatful to them for being nice victims. We thank Robert Kowalski, David S. Warren, Maurizio Martelli, Lee Naish and an anonymous referee for many interesting comments. We thank Wim Vanhoof for encouragements. We thank the organizers of the Shakerstown meeting for their initiative. Danny De Schreye is a senior research associate of the Flemish Fund for Scientific Research (FWO). Marc Denecker is supported by GOA "LP+: a second generation Logic Programming language" and by FWO "Knowledge representation and reasoning in Open Logic Programming".

\section{References}

1. K.R. Apt and R.N. Bol. Logic Programming and negation: a survey. The Journal of Logic Programming, 19-20:9-72, 1994.

2. S. Van Baelen, J. Lewi, and E. Steegmans. Constraints in object-oriented analysis and design. In B. Magnusson, B. Meyer, J.-M. Nerson, and J.-F. Perrot, editors, Technology of Object-Oriented Languages and Systems TOOLS 13, pages 185-199. Prentice-Hall, 1994.

3. M. Bruynooghe, S. Debray, M. Hermenegildo, and M. Maher, editors. The Journal of Logic Programming, Tenth Anniversary Issue, Vol. 19/20. Elsevier Science Publishers, 1994. 
4. A. Bundy. A broader interpretation of Logic in Logic Programming. In K. Bowen and R. Kowalski, editors, Proceedings of the fifth International Conference and Symposium on Logic Programming, pages 1624-1648. MIT-press, 1988.

5. Y. Deville. Logic Programming: Systematic Program Development. AddisonWesley, 1990.

6. Y. Deville and K.-K. Lau. Logic Program synthesis. The Journal of Logic Programming, 19-20:321-350, 1994.

7. R. Glueck and M. H. Soerensen. Partial deduction and driving are equivalent. In M. Hermenegildo and J. Penjam, editors, Programming Languages: Implementations, Logics and Programs, pages 165-181. Springer-Verlag, Lecture Notes in Computer Science, Volume 844, 1994.

8. M. Hermenegildo, G. Puebla, and F. Bueno. Using global analysis, partial specifications, and an extensible assertion language for program validation and debugging. In K.R. Apt, V. Marek, M. Truszczynski, and D.S. Warren, editors, The Logic Programming Paradigm: a 25 Years Perspective (this book). SpringerVerlag, Artificial Intelligence Series, 1999.

9. J. Jaffar and M. Maher. Constraint Logic Programming: a survey. The Journal of Logic Programming, 19-20:503-582, 1994.

10. A. C. Kakas, R. Kowalski, and F. Toni. The role of abduction in Logic Programming. In D. Gabbay, C.J. Hogger, and J.A. Robinson, editors, Handbook of Logic in Artificial Intelligence and Logic Programming 5, pages 235-324. Oxford University Press, 1998.

11. R.A. Kowalski. Algorithm $=$ logic + control. Communications of the ACM, 22:424-431, 1979.

12. R.A. Kowalski. Problems and promises of Computational Logic. In J.W. Lloyd, editor, Symposium on Computational Logic, pages 1-36. Springer-Verlag, 1990.

13. R.A. Kowalski. Logic without model theory. In D. Gabbay, editor, What is a logical system? Oxford University Press, 1995.

14. R. Manthey and F. Bry. A hyperresolution-based proof procedure and its implementation in Prolog. In Proceedings of the 11th German Workshop on Artifial Intelligence, pages 221-230, 1987.

15. S. Muggleton and L. De Raedt. Inductive Logic Programming: theory and methods. The Journal of Logic Programming, 19-20:629-680, 1994.

16. L. Naish. Automating control for Logic Programs. The Journal of Logic Programming, 2(3):167-183, 1985.

17. D. Pearce. Report on the CLN strategic planning workshop, Rome, June 20-21. Technical report, Compulog Network of Excellence, available at http://www.cs.ucy.ac.cy/compulog/newpage5.htm, 1997.

18. T. Le Provost and M. Wallace. Constraint satisfaction over the CLP scheme. The Journal of Logic Programming, Special Issue on Constraint Logic Programming, 16(3-4):319-359, 1993.

19. G. Puebla and M. Hermenegildo. Abstract multiple specialization and its application to program parallelization. Journal of Logic Programming. Special Issue on Synthesis, Transformation and Analysis of Logic Programs, 1999. To appear.

20. Peter Van Roy. The wonder years of sequencial Prolog implementation. The Journal of Logic Programming, 19-20:395-442, 1994. 
21. Konstantinos Sagonas, Terrance Swift, and David S. Warren. XSB as an efficient deductive database engine. In Proceedings of the ACM SIGMOD International Conference on the Management of Data, pages 442-453, Minneapolis, Minnesota, May 1994. ACM Press.

22. D. De Schreye, M. Bruynooghe, B. Demoen, M. Denecker, B. Martens, G. Janssens, L. De Raedt, S. Decorte, M. Leuschel, and K. Sagonas. Lp+: a second generation Logic Programming language. Technical report, Department of Computer Science, K.U.Leuven, available from ftp.cs.kuleuven.ac.be as /pub/dtai/project.ps, 1997.

23. D. A. Smith. Synthesis in the KIDS system. In Y. Deville, editor, Proceedings of the Third International Workshop on Logic Program Synthesis and Transformation. 1993.

24. Zoltan Somogyi, Fergus Henderson, and Thomas Conway. The execution algorithm of Mercury, an efficient purely declarative logic programming language. The Journal of Logic Programming, 29(1-3):17-64, October-December 1996.

25. V. F. Turchin. The concept of a supercompiler. ACM Transactions on Programming Languages and Systems, 8(3):292-325, 1986.

26. David S. Warren. Memoing for Logic Programs. Communications of the ACM, 35(3):93-111, March 1992. 